
\title{
$\angle$ Research Square \\ The Effect of Anesthesia Depth on Radiofrequency Catheter Ablation of Ventricular Tachycardia: A Retrospective Study
}

\section{Hongquan Dong}

Jiangsu Province Hospital and Nanjing Medical University First Affiliated Hospital

\section{Nana Li}

Jiangsu Province Hospital and Nanjing Medical University First Affiliated Hospital

Zhaochu Sun ( $\square$ suncc520@163.com )

Jiangsu Province Hospital and Nanjing Medical University First Affiliated Hospital Department of Anesthesiology https://orcid.org/0000-0002-8663-3321

\section{Research article}

Keywords: Anesthesia depth, Bispectral index, Ventricular tachycardia, Radiofrequency catheter ablation

Posted Date: September 25th, 2020

DOl: https://doi.org/10.21203/rs.3.rs-73623/v1

License: (a) (1) This work is licensed under a Creative Commons Attribution 4.0 International License. Read Full License

Version of Record: A version of this preprint was published at BMC Anesthesiology on November 15th, 2021. See the published version at https://doi.org/10.1186/s12871-021-01503-6. 


\section{Abstract}

Background: Radiofrequency catheter ablation (RFCA) as a safe and effective method has been widely used in ventricular tachycardia (VT) patients, and with which anesthesiologists frequently manage their perioperative care. The aim of this study was to investigate the effects of different anesthetic depths on perioperative RFCA and recurrence in patients who with intractable VT and could not tolerate an awake procedure.

Methods: We reviewed electronic medical records of patients with VT who underwent RFCA by general anesthesia from January 2014 to March 2019. According to intraoperative VT induction, they were divided into two groups: non-inducible group (group N) and inducible group (group I). We constructed several multivariable regression models, in which covariates included patient characteristics, comorbidities, protopathy and BIS value.

Results: 101 patients were analyzed. 29 patients (28.7\%) experienced VT no induction, and 26 patients (25.7\%) recurrented within one year. Compared with group I, the proportion of patients with arrhythmogenic right ventricular cardiomyopathy in group $\mathrm{NI}$ were higher $(P<0.05)$, and the recurrence rate of VT was significantly higher $(51.7 \%$ vs $15.3 \%)(P<0.05)$. Based on prespecified bispectral index (BIS), the BIS $<40$ was associated with elevated odds of VT no induction compared with a BIS value $>50$ (odds ratio, $6.92 ; 95 \%$ confidence interval, $1.47-32.56 ; P=0.01$ ). VT no induction was an independent predictor of recurrence after RFCA (odds ratio, 5.01; 95\% confidence interval, 1.88-13.83; $\mathrm{P}<0.01)$.

Conclusion: Lower BIS value during VT induction in RFCA operation was associated with high risk of VT no induction, which affects postoperative outcomes. We proposed that appropriate depth of anesthesia should be maintained during the process of VT induction.

\section{Trial registration}

ChiCTR, ChiCTR2000034210. Registered 28 June 2020 - Retrospectively registered, http://www.chictr.org.cn/edit.aspx?pid=55691\&htm=4

\section{Background}

Ventricular tachycardia (VT) typically arises from structural heart disease, and increases the risk of sudden cardiac arrests in patients with organic heart disease ${ }^{[1-2]}$. With the deepening understanding of the pathogenesis of ventricular arrhythmia, radiofrequency catheter ablation (RFCA) has become a firstline treatment for refractory $\mathrm{VT}^{[3]}$.

Due to the good sedative and analgesic effects, general anesthesia has been used in RFCA for more complex VT. However, current studies have found that some anesthetics may have the effects of 
myocardial protection and anti-arrhythmia, which might affect cardiac conduction and interfere with the clinical inducibility of $\mathrm{VT}^{[4]}$.

This study aims to compare the effects of different anesthesia depths on the inducibility of VT during RFCA and the postoperative recurrence, which can guide the anesthesiologists to use anesthetics reasonably and help the patients to get through the challenges safely.

\section{Methods}

This retrospective observational study was approved by the Institutional Review Board (IRB) of Jiangsu Province Hospital (JSPH; Jiangsu, China; IRB approval number: 2019-SR-317). Considering the retrospective design of this study, the requirement for informed consent was waived by the IRB.

\section{Data registry and patient selection}

This study utilised data stored and managed in the electronic medical record system of JSPH on the adult patients (18 year) who were decided to perform RFCA operation under general anesthesia by cardiologist due to VT, between January 2014 and Apirl 2019 (Fig. 1). All the cases for the study period were screened by a group of medical record technicians in the medical informatics team who were not informed of the purpose of this study. Patients who were lost to follow-up within one year after surgery, were excluded from the analysis.

To compare the relationship between the success rate of VT inducibility and gradient of intraoperative BIS, we divided recipients into two groups as follows: non-inducible group (group N) and inducible group (group I). We hypothesized that the BIS values were associated with VT inducibility, which might affect the procedure of operation and recurrence. The primary outcome was assessed by comparing the induction rate and the gradient of intraoperative bispectral index (BIS) between the two groups. We recorded the selected information: gender, age, primary disease, cardiac function, comorbidities; the BIS during VT induction in the two groups, as well as the operative time, ablation time (from the beginning of mapping to the end of ablation), and fluoroscopy time. Patients were followed up regularly for one year after the operation, and the recurrence was recorded.

\section{Monitoring and Anesthesia}

All operations were performed using standard VT techniques, and intraoperative anesthetic management was performed with continuous monitoring of peripheral capillary oxygen saturation, electrocardiography (ECG), arterial blood pressure. Anesthesia was induced with midazolam $0.05 \mathrm{mg} / \mathrm{kg}$, fentanyl $3 \mathrm{mg} / \mathrm{kg}$, propofol $2 \mathrm{mg} / \mathrm{kg}$, cisatracurium $0.15 \mathrm{mg} / \mathrm{kg}$. The BIS (Aspect Medical System, Inc, Norwood, Mass, United States) sensor was applied to the patient's forehead after anesthesia induction, and the BIS was monitored throughout the RFCA procedure. Sevoflurane and propofol were used for anesthesia maintenance, and cisatracurium for muscle relaxation. The depth of anesthesia was guided by the hemodynamic parameter and BIS to prevent intraoperative awareness. 


\section{Electrophysiological mapping and radiofrequency ablation}

VT induction

Quadrupole electrode was first used for program stimulation at specific stimulation sites. When VT was not induced, Burst stimulation was performed. In case that VT still wasn't induced, isoproterenol would be given to increase the heart rate by about $20 \%$ and then the above stimulation would be repeated until VT was induced. When all the steps were completed and repeated for three times but it could not be induced, it would be defined as VT no induction.

VT mapping

In stable VT, activation and entrainment mapping during VT are used to find the crucial circuit that will guide ablation. For multiple and unstable VTs, pace mapping can reveal scarred regions and potential reentry-circuit channels during sinus or paced rhythm.

VT ablation

We used temperature control and non-saline perfusion ablation strategy for VT. After VT disappeared, we observed for 30 minutes. Then, ventricular program pre-phase stimulation and graded increasing stimulation were performed under conditions of basic state and isoproterenol intravenous injection. When the right ventricular outflow tract ventricular contraction or VT can not be induced, it is regarded as the succeed of immediate ablation.

Postoperative follow-up

On the second day after ablation, the patients underwent a Dynamic Electrocardiography (DCG). ECG and DCG were reviewed at 3rd, 6th and 12th months postoperative sessions, and the symptoms of VT recurrence were checked by cardiac electrophysiologist to comprehensively determine whether there was VT recurrence.

\section{Statistical Analysis}

Statistical analysis was performed with SPSS 20.0 (IBM Software Inc., USA). Categorical variables were presented using numbers with percentages and were analyzed with chi-square test or Fisher's exact test, whereas the continuous variables were expressed as mean \pm standard deviation, and were compared with the Student's t-test for unpaired samples when a normal deviation was assumed. Univariate and stepwise multivariate logistic regression analysis were performed to determine the risk factors of VT no induction and VT recurrence. All clinically sensible covariates were included in the model. For all analysis, a $P$ value of $<0.05$ was considered statistically significant.

\section{Results}


We identified a total of 101 patients meeting the inclusion criteria during the study period. The mean age of all patients was $48.7 \pm 16.2$ years, and the mean LVEF was $53.6 \pm 11.7 \%$ in the baseline echocardiogram. Twenty two (21.8\%) patients had a history of catheter ablation, and twenty six $(25.7 \%)$ experienced the recurrence within one year. Patient demographic characteristics are shown in Table 1. There were no differences with baseline characteristics before operation between the two groups. However, the protopathy, BIS values and VT recurrence were different, with more ARVC patients $(P=0.03)$, lower BIS values $(P<0.01)$ and higher recurrence rate of VT $(P<0.01)$ in group N (Table 1$)$.

The radiation time demonstrated no significant difference between the two groups; however, the difference with operative time and ablation time in group $\mathrm{N}$ remained significant longer than those in group I $(P<0.05)$ (Table 2$)$.

Multivariate logistic regression analysis identified that ARVC (OR, 3.17; 95\% Cl, 1.23-8.15; $P=0.02)$ and BIS value $<40(\mathrm{OR}, 6.92 ; 95 \% \mathrm{Cl}, 1.47-32.56 ; P=0.01)$ were associated with VT induction (Table 3$)$. In addition, VT no induction was an independent risk factor (OR, 5.01; $95 \% \mathrm{Cl}, 1.88-13.83 ; P<0.01)$ for the VT recurrence within one year (Table 4).

Figure 1. Flowchart summarising the steps used for patient selection. 
Table 1

Baseline characteristics of 101 patients with a diagnosis of VT and who received RFCA under general anesthesia. Values are number (proportion) or mean (SD).

\begin{tabular}{|llll|}
\hline & Group N(n= 29) & Group I(n= 72) & PValue \\
\hline Age & $45.9(14.1)$ & $49.9(16.9)$ & 0.27 \\
\hline Gender (M/F) & $27 / 2$ & $64 / 8$ & 0.79 \\
\hline BMI $\left(\mathrm{Kg} / \mathrm{m}^{2}\right)$ & $24.6(2.1)$ & $23.8(2.5)$ & 0.13 \\
\hline LVEF & $56.3(11.3)$ & $52.5(11.7)$ & 0.14 \\
\hline Comorbidity & & & 0.46 \\
\hline Hypertension & $6(20.7 \%)$ & $20(27.8 \%)$ & 0.91 \\
\hline Diabetes mellitus & $3(10.3 \%)$ & $8(11.1 \%)$ & 0.22 \\
\hline Coronary artery disease & $2(6.9 \%)$ & $13(18.1 \%)$ & 0.37 \\
\hline Protopathy & & $24(33.3 \%)$ & $0.03 *$ \\
\hline Dilated cardiomyopathy & $7(24.1 \%)$ & $25(34.7 \%)$ & 0.16 \\
\hline ARVC & $17(58.6 \%)$ & $41(56.9 \%)$ & 0.29 \\
\hline ICD implantation & $12(41.4 \%)$ & $18(25 \%)$ & $<0.01^{*}$ \\
\hline History of VT ablation & $4(13.8 \%)$ & $51.5(6.1)$ & $<0.01^{*}$ \\
\hline BIS & $46.2(8.6)$ & $11(15.3 \%)$ & \\
\hline Recurrence within 1 year & $15(51.7 \%)$ & & \\
\hline $\begin{array}{l}\text { Abbreviations: BMI, body mass index; LVEF, left ventricular ejection fraction; ARVC, arrhythmogenic } \\
\text { right ventricular cardiomyopathy; ICD, implantable cardioverter-defibrillator; VT, ventricular } \\
\text { tachycardia; BIS, bispectral index. }\end{array}$ & & & \\
\hline * $P<0$ 05. & & & \\
\hline
\end{tabular}

Table 2

Outcome data of surgical indicators between group A and C, mean and standard deviation are displayed.

\begin{tabular}{|llll|}
\hline & Group N(n= 29) & Group I(n= 72) & $P$ Value \\
\hline Operative time (min & $241.6(49.5)$ & $219.2(42.4)$ & $0.02^{\star}$ \\
\hline Radiation time (min) & $18.2(8.1)$ & $19.9(6.4)$ & 0.26 \\
\hline Ablation time (min) & $156.0(28.7)$ & $141.4(25.8)$ & $0.01^{\star}$ \\
\hline$* P<0.05$. & & & \\
\hline
\end{tabular}


Table 3

Univariate and multivariate logistic regression analysis about VT induction during RFCA.

\begin{tabular}{|c|c|c|c|c|}
\hline & Univariable & & Multivariable & \\
\hline Variables & OR $(95 \% \mathrm{Cl})$ & $P$ Value & OR $(95 \% \mathrm{Cl})$ & $P$ Value \\
\hline Age & $1.02(0.99-1.04)$ & 0.27 & - & - \\
\hline Gender (M) & $1.93(0.39-9.53)$ & 0.42 & - & - \\
\hline LVEF & $0.97(0.93-1.01)$ & 0.14 & - & - \\
\hline \multicolumn{5}{|l|}{ Protopathy } \\
\hline Dilated cardiomyopathy & $1.57(0.59-4.19)$ & 0.37 & - & - \\
\hline ARVC & $2.67(1.10-6.45)$ & $0.03^{*}+$ & $3.17(1.23-8.15)$ & $0.02^{*}$ \\
\hline History of VT ablation & $2.08(0.64-6.80)$ & 0.22 & - & - \\
\hline \multicolumn{5}{|l|}{ BIS } \\
\hline$>50$ & 1 & - & 1 & - \\
\hline $40-50$ & $1.38(0.53-3.62)$ & $0.51 \dagger$ & $1.74(0.63-4.80)$ & 0.29 \\
\hline$<40$ & $5.33(1.23-23.01)$ & $0.03^{\star}+$ & $6.92(1.47-32.56)$ & $0.01^{*}$ \\
\hline Recurrence within 1 year & $5.94(2.25-15.69)$ & $<0.01^{*}$ & $5.01(1.88,13.83)$ & $<0.01^{\star}$ \\
\hline \multicolumn{5}{|c|}{$\begin{array}{l}\text { Abbreviations: LVEF, left ventricular ejection fraction; ARVC, arrhythmogenic right ventricular } \\
\text { cardiomyopathy; VT, ventricular tachycardia; BIS, bispectral index. }\end{array}$} \\
\hline \multicolumn{5}{|l|}{$\star P<0.05$} \\
\hline † Analyzed using multiva & inalysis. & & & \\
\hline
\end{tabular}


Table 4

Univariate and multivariate logistic regression analysis about the recurrence of VT after RFCA.

\begin{tabular}{|c|c|c|c|c|}
\hline & Univariable & & Multivariable & \\
\hline Variables & OR $(95 \% \mathrm{Cl})$ & $P$ Value & $\mathrm{OR}(95 \% \mathrm{Cl})$ & $P$ Value \\
\hline Age & $0.99(0.97,1.02)$ & 0.69 & & \\
\hline Gender (M) & $3.57(0.43,29.67)$ & 0.24 & & \\
\hline LVEF & $0.99(0.96,1.03)$ & 0.71 & & \\
\hline \multicolumn{5}{|l|}{ Protopathy } \\
\hline Dilated cardiomyopathy & $0.66(0.26,1.69)$ & 0.38 & & \\
\hline ARVC & $1.43(0.58,3.54)$ & 0.44 & & \\
\hline ICD implantation & $0.96(0.39,2.35)$ & 0.93 & & \\
\hline History of VT ablation & $2.56(0.69,9.53)$ & 0.16 & & \\
\hline \multicolumn{5}{|l|}{ BIS } \\
\hline$>50$ & 1 & & 1 & \\
\hline $40-50$ & $1.30(0.48,3.57)$ & $0.61 \dagger$ & $1.19(0.41,3.45)$ & 0.75 \\
\hline$<40$ & $6.19(1.41,27.24)$ & $0.02 * \dagger$ & $4.01(0.81,19.84)$ & 0.09 \\
\hline Non-induction & $5.94(2.25,15.69)$ & $<0.01 * \dagger$ & $5.01(1.88,13.83)$ & $<0.01^{*}$ \\
\hline \multicolumn{5}{|c|}{$\begin{array}{l}\text { Abbreviations: LVEF, left ventricular ejection fraction; ARVC, arrhythmogenic right ventricular } \\
\text { cardiomyopathy; ICD, implantable cardioverter-defibrillator; VT, ventricular tachycardia; BIS, bispectra } \\
\text { index. }\end{array}$} \\
\hline \multicolumn{5}{|l|}{$\star P<0.05}$. \\
\hline † Analyzed using multiva & analysis. & & & \\
\hline
\end{tabular}

\section{Discussion}

RFCA is widely used to manage VT associated with structural heart disease when implantable cardioverter defibrillator (ICDs) or antiarrhythmic drugs have failed, and it is usually the sole treatment for idiopathic $\mathrm{VT}^{[5]}$. However, the effect is still unsatisfactory. Some studies have showed that one-year success rate after catheter ablation is $70 \%[6]$, and the 5 -year recurrence rate is still as high as $46 \%{ }^{[7]}$. In this retrospective study, 101 patients who with intractable VT and operated under general anesthesia were included. Among them, 29 patients(28.7\%) experienced VT no induction during operation, and 26 patients $(25.7 \%)$ relapsed within one year. This study also demonstrated that the lower BIS value $<40$ was one risk factor for VT no induction, what's more, the recurrence rate of VT was significantly higher in 
group N than group I (51.7\% vs $15.3 \%)$, which shows VT no induction was an independent predictor of VT recurrence within one year.

Currently, wake-state or MAC is the most commonly used anesthetic method for RFCA. However, for more complex VT and unstable patients, general anesthesia is still required. Regular mechanical ventilation can reduce the interference of breathing on RFCA, improve the success rate of operation, shorten the operation time, reduce the recurrence rate and adverse reactions ${ }^{[8]}$. Unfortunately, clinical practice has shown that general anesthesia may cause VT induced failure or hemodynamic instability. The inducibility of arrhythmia is important, much ablation for ventricular arrhythmia targets symptomatic focal PVCs rather than sustained VT. Furthermore, activation and entrainment mapping, and searching for the earliest PP are still the most widely used techniques ${ }^{[9-10]}$, which can only be used when VT induced stability. Therefore, for those patients who cannot be induced, it is often difficult to map the ablation target, thus affecting the success rate of ablation.

Many anesthetics (such as inhaled anesthetics, propofol and dexmedetomidine, etc.) have been shown their potential effects on cardiac conduction and interfere with the clinical induction of VT, whatsmore, some types of VT (such as outflow tract VT caused by ARVC) are extremely sensitive to sedation ${ }^{[3]}$. Therefore, to control the depth of anesthesia and to ensure the induction of arrhythmia and hemodynamic stability plays a very important role in the outcome of VT treatment.

Propofol is widely used during the induction and maintenance of anesthesia. Apart from these anesthetic properties, propofol has additional antiarrhythmic and proarrhythmic effects. At clinically relevant concentrations, the incidence of arrhythmia is relatively low, but the effect of arrhythmia inhibition is more significant. Studies have demonstrated that propofol has a protective effect on myocardial ischemia and arrhythmia which are caused by ischemia-reperfusion injury ${ }^{[11-12]}$. Propofol also has been shown to terminate atrial fibrillation and VT storm ${ }^{[13-14]}$. This effect may be due to the fact that the electrical storms are often caused by adrenergic stimulation, what's more, propofol can reduce the sympathetic tension by mediated GABA receptor, and inhibit sympathetic activity and cardiac electrical storm $^{[15-16]}$. In addition, propofol can also shorten the Q-T interval of long Q-T syndrome; therefore, it may have the potential to prevent episodes of VT which are caused by Q-T interval dispersion ${ }^{[17]}$.

Similarly, sevoflurane may also affect the induction of $\mathrm{VT}$, which mainly produces a cascade reaction through a variety of signal transduction pathways such as protein kinase $\mathrm{C}$, tyrosine protein kinase, etc., changing the gene expression of cardiomyocytes, and producing a variety of anti-injury factors. Furthermore, sevoflurane also prevents intracellular calcium overload through KATP channels, reduces ischemia-reperfusion injury and produces cellular protection ${ }^{[12]}$, so as to decrease the occurrence of ventricular arrhythmias. In addition, sevoflurane and other volatile anesthetics can also delay atrioventricular repolarization and reduce the possibility of inducing ventricular arrhythmia in vitro by prolonging the duration of the action potential, but its clinical significance is not clear. 
Recently, some experts have suggested that monitored anesthesia care (MAC) can be used to replace traditional general anesthesia in RFCA, which can effectively reduce the use of anesthetics that may affect VT induction and ensure the hemodynamics stability. However, MAC also has its limitations, which may increase the risk of respiratory depression and hypoxia. Therefore, the choice of the ideal anesthesia program still needs to weigh the pros and cons.

Our study has several limitations. First, this study is a retrospective study, in which only BIS values were used as the evaluation index for anesthesia depth. It is difficult to track the real-time blood concentration of various anesthetics, and it is impossible to determine which drug is the main factor that causes noninducible of VT. Our team will conduct a prospective group analysis of the influence factors in the followup prospective research. Second, the number of cases in this study is small and it is a single-center study, which may lead to the bias of results and a large $95 \% \mathrm{Cl}$.

In conclusion, we have reported that patients with VT who experience RFCA under general anesthesia, with the increase of anesthesia depth, may increase the risk of VT no induction, which in turn affects postoperative outcomes. Anesthesiologists need to have a deeper understanding of the effects of anesthesia methods and drugs on RFCA, so as to choose the ideal anesthesia management strategy and anesthesia depth to ensure the patient safety while improving the success rate of surgery.

\section{Declarations}

\section{Acknowledgements}

This study was supported by the department of anesthesia and cardiology, Nanjing Medical University.

\section{Availability of data and materials}

The datasets used and/or analyzed during this study are available from the corresponding author on reasonable.

\section{Authors' contributions}

Zhaochu Sun designed the study and wrote the manuscript. Hongquan Dong, Nana Li collected the data, analyzed the data, and interpretation of data. All authors revised the manuscript critically for important intellectual content. All authors read and approved the final manuscript.

\section{Ethics approval and consent to participate}

This retrospective study was approved by the Institutional Review Board of Jiangsu Province Hospital (JSPH 2019-SR-317) and the requirement for written informed consent was waived.

\section{Consent for publication}

Not applicable. 


\section{Competing interests}

The authors declare that they have no competing interests.

\section{Funding}

National Natural Science Foundation of China (81801061).

\section{References}

1. Priori SG, Blomström-Lundqvist C, Mazzanti A,et al. 2015 ESC guidelines for the management of patients with ven- tricular arrhythmias and the prevention of sudden cardiac death. Eur Heart $\mathrm{J}$. 2015;36:2793-867.

2. Al-Khatib SM, Stevenson WG, Ackerman MJ, et al. 2017 AHA/ACC/HRS guideline for management of patients with ventricular arrhythmias and the prevention of sudden cardiac death: a report of the American College of Cardiology/American Heart Association Task Force on Clinical Practice Guidelines and the Heart Rhythm Society. Heart Rhythm. 2018;15:e190-252.

3. Deng Y, Naeini PS, Razavi M, et al. Anesthetic Management in Radiofrequency Catheter Ablation of Ventricular Tachycardia. Tex Heart Inst J. 2016;43:496-502.

4. Haqqani HM, Roberts-Thomson KC. Radiofrequency catheter ablation for ventricular tachycardia. Heart Lung Circulation. 2012;21:402-12.

5. Pedersen CT, Kay GN, Kalman J, et al. EHRA/HRS/APHRS expert consensus on ventricular arrhythmias. Heart Rhythm. 2014;11:e166-96.

6. Tung $\mathrm{R}$, Vaseghi M, Frankel DS, et al. Freedom from recurrent ventricular tachycardia after catheter ablation is associated with improved survival in patients with structural heart disease:an International VT Ablation Center Collaborative Group study. Heart Rhythm. 2015;12:1997-2007.

7. Donateo P, Bottoni N, Oddone D, et al. Long-Term Results After Single and Multiple Procedures of Ablation of Ventricular Tachycardia. J Cardiovasc Electrophysiol. 2016;27:1319-24.

8. Du XH, Wang X. OPLER with sevoflurane laryngeal mask airway in general anesthesia in interventional operation. Journal of Medicine Forum. 2011;30:111-14.

9. Sung R, Scheinman M. Spectrum of Fascicular Arrhythmias. Cardiac Electrophysiology Clinics. 2016;8:567-80.

10. Liu Y, Fang Z, Yang B, et al. Catheter Ablation of Fascicular Ventricular Tachycardia: Long-Term Clinical Outcomes and Mechanisms of Recurrence. Circulation Arrhythmia Electrophysiology. 2015;8:1442-51.

11. Liu Q, Chen R, Hong J, et al. Propofol and arrhythmias: two sides of the coin. Acta Pharmacol Sin. 2011;32:817-23.

12. Yang $X L$, Wang D, Zhang GY, et al. Comparison of the myocardial protective effect of sevoflurane versus propofol in patients undergoing heart valve replacement surgery with cardiopulmonary 
bypass. BMC Anesthesiol. 2017;1:37-44.

13. Miro $\mathrm{O}$, de la Red G, Fontanals J. Cessation of paroxysmal atrial fibrillation during acute intravenous propofol administration. Anesthesiology. 2000;92:910.

14. Mulpuru SK, Patel DV, Wilbur SL, et al. Electrical storm and termination with propofol therapy: a case report. Int J Cardiol. 2008;128:e6-8.

15. Dan S, Thomas MM, Shen WK. Optimal antiarrhythmic drug therapy for electrical storm. Journal of Biomedical Research. 2015;29:20-34.

16. Molina PE. Opioids and opiates: analgesia with cardiovascular, haemodynamic and immune implications in critical illness. J Intern Med. 2006;259:138-54.

17. Kleinsasser $\mathrm{A}$, Loeckinger $\mathrm{A}$, Lindner $\mathrm{KH}$, et al. Reversing sevoflurane-associated Q-Tc prolongation by changing to propofol. Anaesthesia. 2001;56:248-50.

\section{Figures}

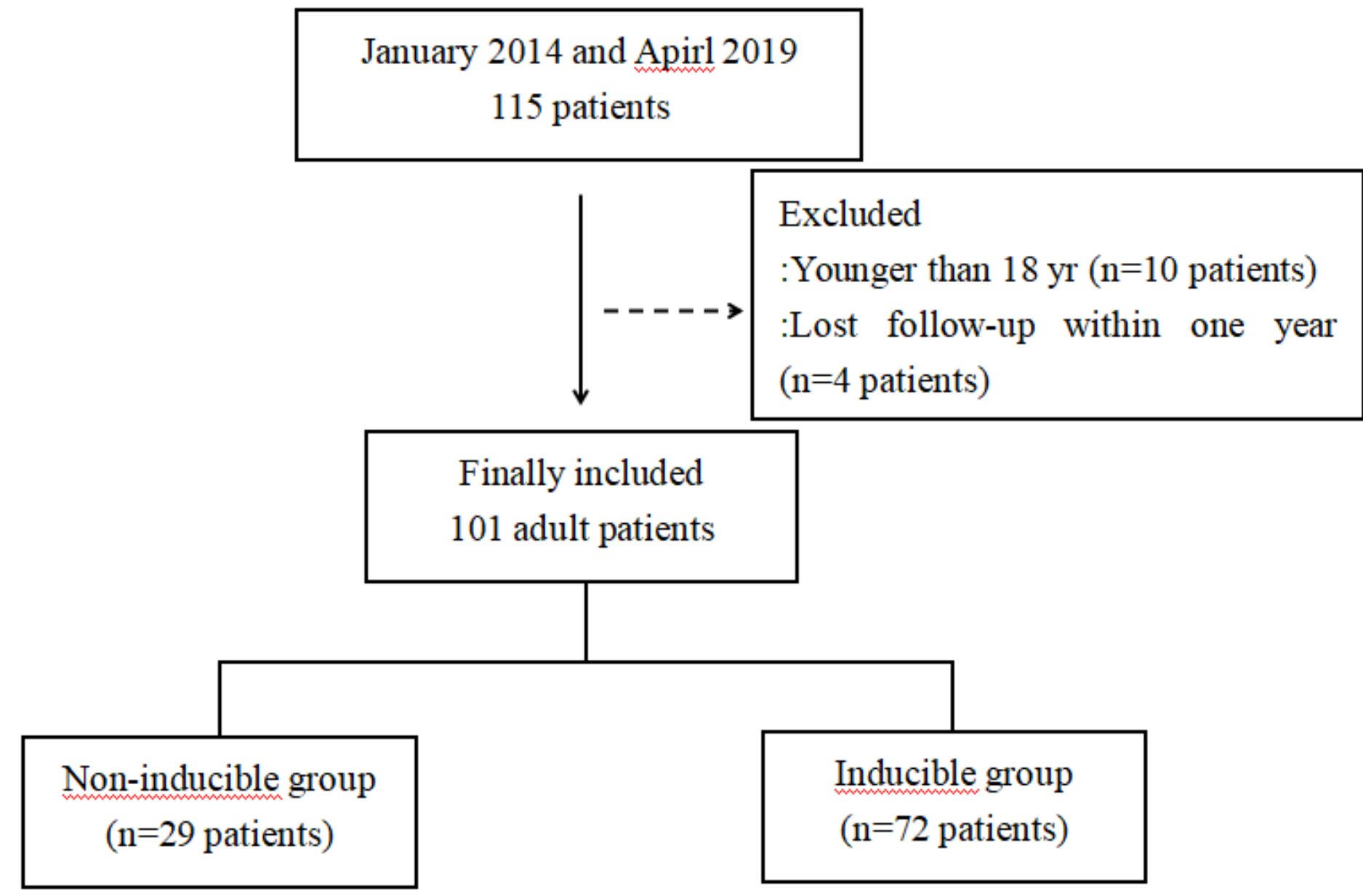

Figure 1

Flowchart summarising the steps used for patient selection. 